\title{
CONSENSUS MAKING IN REQUIREMENTS NEGOTIATION: THE COMMUNICATION PERSPECTIVE
}

\author{
Jenny Price \\ jpr@deakin.edu.au \\ Jacob L. Cybulski \\ jlcybuls@deakin.edu.au \\ +61392446847 \\ School of Information Systems \\ Deakin University \\ 221 Burwood Highway \\ Burwood, Victoria 3125, Australia
}

\begin{abstract}
When developing an Information System (IS), organizational goals of various stakeholders are commonly in direct conflict. Furthermore, individuals often rank their private objectives well over their management's directions. Recognising and reconciling all these diverse goals, and reaching agreement among the stakeholders, are prerequisite to establishing project cooperation and collaboration. This paper focuses, in particular, on the negotiation and consensus making during requirements elicitation - the earliest stages of the IS development process. As requirements elicitation involves rich communication between project stakeholders, we therefore explore negotiation and consensus making from the communication perspective. The resulting model assists our understanding of the communication factors that influence the consensus process during requirements negotiation.
\end{abstract}

\section{INTRODUCTION}

Requirements Engineering (RE) is a cooperative learning process where stakeholders from different backgrounds with different experiences and objectives have to communicate to elicit and validate requirement (Bustard 2002). Not surprisingly this stakeholder interaction as a group provides an opportunity for the emergence of misunderstanding and conflict (Eastbrook 1993, Sommerville \& Kotonya 1996, Weigers 2000). The catalyst being that even when common objectives are set, agreed to and acknowledged by others, the participants often misinterpret objectives due to the influence of their bias, personality and environment (Eastbrook 1993, Sommerville \& Kotonya 1996, Weigers 2000. Therefore alignment of viewpoints may be necessary to prevent conflict and its associated problems (Easterbrook 1994).

In the past (Sommerville \& Kotonya 1996, Finkelstein \& Sommerville 1994), it has been argued that viewpoints reconciliation could be achieved by means of their representation in a formal notation and the subsequent formalism manipulation. In our research, however, we take a different view of consensus building, essentially that it is a social process of communicative actions (Cecez-Kecmanovic \& Janson 1999, Cecez-Kecmanovic 2001). This paper further argues that consensus and the associated alignment of stakeholder viewpoints involves not only communication between individuals involved in the IS project but also that it relies on the processes associated with the setting of management and organisational goals. Thus the ensuing discussion will ultimately lead to the development of a model (see Figure 1) representing the communication factors that influence stakeholder requirements negotiation and the consensus making process - the IC Model. 


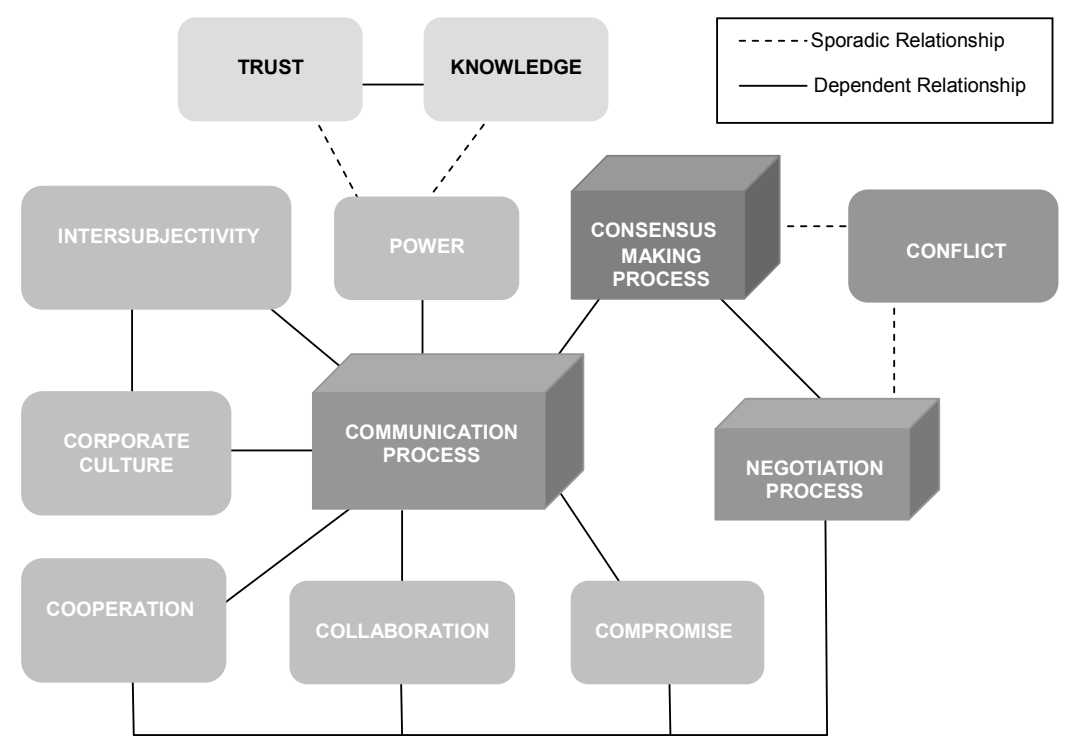

Figure 1 - The Initial Model of Influences on Consensus (IC) (As resulted from this study

\section{Consensus}

The consensus making process has been defined as a group process where stakeholder input is carefully considered so an outcome that best meets the needs of the group can be crafted (Deutsch 1973, Dessein 2002, Davis et al 2000, Darke \& Shanks 1997). Surprisingly, consensus does not require unanimity but in order for all participants to the process to accept the decision they must feel that their perspectives and ideas are acknowledged to allow the cooperative dynamic to be created (Cecez-Kecmanovic \& Janson 1999). However at this point it must be noted that consensus outcomes do not necessarily involve negotiation as a consensus outcome can be manipulated subsequently changing the state of the consensus from 'natural' where all parties have of their own accord, agreed to an acceptable outcome, to 'forced' where some of the stakeholders have been openly or surreptitiously coerced into agreement. Following is a discussion of the communication factors that can influence the consensus process during requirements negotiation.

\section{The Negotiation Process}

In respect to the RE field we understand that consensus outcomes not achieved through negotiation can lead to stakeholder dissatisfaction and non-acceptance of information systems (Coombes 2001). Consensus doesn't occur without some active intervention and bargaining of the participating parties; instead it is commonly negotiated (Weigers 2000). And so, the process of negotiation is deemed as having a direct affect on the consensus process, while it also has a natural affect on the emerging conflicts (see Figure 1). Negotiating in conflict situations is considered one of the main tools to facilitate 
satisfactory conclusion to the entire cycle of RE (Weigers 2000), and it may require some compromises on behalf of the participants (Bustard 2002). It should be noted that consensus normally calls upon negotiation tactics, which when successful may avert conflict situations.

Lewicki et al (1999) examined various aspects of negotiation concluding that it consists of at least four typical elements.

Conflict is recognised as the first and the foremost element in negotiation. They argue that conflict situations may, in fact, influence stakeholder expectations of possible negotiation outcomes and fairness in settlement. Settlements are commonly achieved through negotiating parties sacrificing their objectives and the expected outcomes. Participants in negotiation are more willing to accept such sacrifice to the benefit of their counterpart if they have a positive relationship with that counterpart. In support, Drolet et al (1998) argued when counterparts are in a negative relationship they tend to risk disadvantageous inequality in order to maximise their own expected outcomes and to increase the likelihood of obtaining advantageous inequality.

The second negotiation element recognised by Lewicki (1999) is a degree of interdependence between the counterparts. They believe that all parties involved in negotiation must be reliant on the other party to some extent or negotiation of an accepted outcome would not be necessary.

The third element is an opportunistic interaction that provides the counterparts the access to influence each other (Lewicki 1999). When negotiating this opportunistic interaction cannot occur without the counterparts communicating in some way. Drolet et al. 1998 clearly acknowledge communication to facilitate cooperation in mixed motive conflicts. They have also demonstrated that the type of communication media used to facilitate negotiation influences the outcomes. In particular, the less a medium allows for synchronous, multiple channel expression of emotion, the less it will foster rapport.

Lewicki et al. (1999) discuss the fourth and final negotiation ingredient being the possibility of agreement. They argue that parties would not even sit at a negotiation table unless they could see the very possibility for reaching an acceptable outcome.

The discussion so far demonstrates the importance of communication to negotiation and therefore the consensus making process. It should also be clear that the decision to adopt an appropriate negotiation strategy could determine the consensus outcome as well.

\section{Conflict}

The initial stage of IS system development, requirements elicitation, provides ample scope for early reconciliation of stakeholders' opinions and thus also opportunities to avoid possible conflict (Somerville \& Kotonya 1996, Wood \& Webb 2002) (see Figure 1). Conflict has been defined as any interference in one party's activities, needs or goals, caused by the activities of another party (Easterbrook 1994). Easterbrook $(1993,1994)$ and Kirsch (2000) believe that conflict is inevitable in requirements elicitation; they feel that conflict is likely to occur because requirements are necessarily negotiated - not captured, as it is commonly believed. They also believe that interdependence among parties and the divergence of interests, opinions or goals amongst the stakeholders contribute to conflict. Several authors (Hancock 2002, Curtis et al 1988, Rothstein \& Butler 1998) have indicated that to achieve consensus, the conflict resolution approach must emphasise communication between participants. This is because consensus is different from other kinds of decisionmaking as it stresses the cooperative development of decision making with group members 
working together rather than competing against each other. For this reason and even though conflict has been included as a factor in the model (see Figure 1) we focus on those communication factors that directly contribute to the negotiation process and thus conflict resolution or consensus making.

\section{Communication}

Indisputably, stakeholder communication is a central element to reaching consensus (sandelin 1999) (see Figure 1). Bovee and Thill (2000) examined the nature of business communication, defining it as a dynamic process involving distortion and interference, and recognising that it has the ability to facilitate and inhibit consensus negotiation. They clearly acknowledge that, in the most general sense, communication involves encoding and decoding of messages by communicative participants. This allows for perceptions, perspectives and interpretations to play part in an individual's understanding or representation of communicated messages (Bovee and Thill 2000). If all parties to the message transmitted do not have exactly the same life experiences, then their individual prior experiences give them distinct cognitive viewpoints (Bovee and Thill 2000, Kaplan \& Martin 1999). Therefore with no regard to external interference, otherwise known as 'noise', it is almost impossible to expect two parties to a message to interpret it in the same way (Bovee and Thill 2000, Kaplan \& Martin 1999).

\section{Intersubjectivity}

Interpretation has been labelled a barrier to communication and a contributor to misunderstanding (Bovee and Thill 2000). It has been reported that when communicating, people use selective perception, distortion or interference which can inhibit the communication (Bovee and Thill 2000). Easterbrook (1994) further acknowledged that interpretation is based on intersubjectivity or 'mutual understanding' which changes with communication and a person's position in a group at the time of communication (see Figure 1). From a sociological point of view Habermas acknowledged that language and socialisation greatly affect a person's opinions and perspectives thus influencing the politics of communication. While applying Habermas' theories to the context of information systems, Cecez-Kecmanovic and Janson (1999) categorise stakeholder communication actions as being:

- 'Oriented to understanding' when stakeholders aim to achieve their goals through 'communicative action'; or

- 'Oriented to success' when stakeholders aim to achieve their goal by intervening in the system or, by influencing others by acting strategically and disregarding others' needs, interests or values.

Expanding further on the social issues, Mills (2003) noted that individuals' interpretation, or 'sense making', of organisational communication is related to their emotional engagement and the prevailing issue climate. Mills (2003) elaborated that individuals engaged emotionally with the discourse commonly use interpretations of shared communication distinct from indifferent individuals. However intersubjectivity can be achieved when the communicating stakeholders share the perception of the topic of conversation (Easterbrook 1994, Yin 1994). In fact, as participants increase their amount of shared experiences, or common ground, they concurrently increase their level of 
understanding of a shared issue (Bovee and Thill 2000). Therefore intersubjectivity, as emerging through the continuum of stakeholders' communication, plays a vital role in the individuals' ability to reach an agreement in the consensus process.

\section{Corporate Culture}

Corporate culture has been defined as a set of norms, beliefs and principles that shape the way an organisation's staff perceive, interpret and communicate with each other resulting in each organisation's distinctive character (Bovee and Thill 2000, Yin 1993). In the workplace, intersubjectivity and the organisation's culture influence each other qualifying this factor as having mutual influence on communication and the consensus process (Bovee and Thill 2000) (see Figure 1).

\section{Compromise, Collaboration \& Cooperation}

Requirements elicitation is considered a profoundly collaborative task that relies on effective communication and interaction between all participating stakeholders (Darke \& Shanks 1997). Therefore stakeholder misperceptions of communicative intentions could ultimately lead to misunderstandings and conflict Kethers (2001). Yet we should not consider good communication the only important influence on cooperative effort Klischewski 2001, Ransley 2000). In fact, projects benefit greatly from good requirements management principles, open and trusting environment, with business, stakeholders and developers working together in a collaborative and cooperative fashion, all to enable the sharing of viewpoints, clarification of conflict and consensus negotiation.

Not surprisingly, in any decision making group participants commonly form distinctive and dissenting factions, even in spite of their shared social status (Kaplan \& Martin 1999). On the other hand, as collaboration requires shared perceptions of all parties involved, this is more likely to come from members of a shared social status (Spekman 1996). Though, it is not unusual for executives, who are usually more familiar with the company's challenges and direction, to negotiate a consensus amongst themselves that could be quite opposite to that of subordinate opinions (Berman \& Werther 1996).

Therefore we can confidently acknowledge that compromise, collaboration and cooperation have all been identified as having an influence on the consensus process directly (see Figure 1). All three factors must be communicated to have influence on consensus therefore a dependent relationship between communication and the three factors will be represented in the IC Model.

\section{Power, Knowledge and Trust.}

Deutsch (1973) recognises power as a direct influence on group decision-making. He further remarks that within the group dynamics, group power is facilitated through normative influence which is a social-relational form of influence in which pressure is applied to deviant members to conform to 'the group norm' (Deutsch 1973). Kaplan and Martin (1999) raised the social status of individuals within the group as another aspect of minority power. Eisenhardt and Bourgeois (1986) and Flood et al. (2000) supported this noting that Chief Executive Officer (CEO) dominance reduces the degree of consensus that is achieved when group decision-making.

Deutsch (1973) also acknowledged informational influence as a source of individual or 
minority power saying that a group member might be influenced to accept third party information as being representative of a specific situation or fact even if that is not the case because of the informational power the influencing party has over them or the group. Kaplan and Martin (1999) supported this recognising expert power as an influence of minority power, defining it as a special knowledge or expertise about an issue that may influence a decision.

Recognising knowledge or expert power as a sub factor of power justifies its inclusion in the IC Model. The power associated with knowledge is limited to situations where there is a disparity in the knowledge level of stakeholders. This will not always be the case therefore the relationship type between knowledge and power will be presented in the IC Model as sporadic (see Figure 1).

Bachmann (1999) raised a further influence on consensus - trust. He stated that trust creates a reduction of possibilities in a decision making process and thus reduces complexity. This equates to trust having influence on the consensus process which has previously been defined as a decision making process. Child and Möllering (2001) believe that trust takes on three forms when it comes to people and technology, i.e. trust in technology, organisational trust, and trust in others in the organisational environment (social trust).

Bachmann (1999) further argued that power and trust are alternative mechanisms to coordinate social interactions efficiently and to allow for relatively stable relationships between co-operating social actors, such as decision making groups. He stated that trust works on the basis of positive assumptions whereas power is based on negative hypothetical possibilities, which is presented by the powerful actor and believed by the subordinate actor to not be in the interest of either side. Even though Bachmann (1999) classified power and trust as alternative mechanisms, he did not exclude them from occurring in combination. In fact he said that power often appears as a precondition rather than as an alternative to trust. Farrell (2002) agreed stating that power and trust are not mutually exclusive. He says that disparities in power affect the way in which the proceeds of trust-based cooperation are distributed, but do not prevent trust from occurring.

The acknowledgement of the association between trust, knowledge and power means that trust will also be included in the IC Model of factors that influence consensus (see Figure 1). Farrell (2002) and Giddens' (1991) descriptions of trust established trust relationship to power, as being sporadic for each can exist without the presence of the other. However the relationship that exists between knowledge and trust must be dependent as the power associated with knowledge is diffused if there is no trust in the knowledge.

\section{The IC Model}

At this point, the review of the extant literature had given us some preliminary insights as to the factors that could possibly influence the negotiation of a consensus and the relationships that may exist between these factors. A conceptual model of Influences on Consensus (IC) (see Figure 1) was thus developed to initially capture the factors and then elaborate and evaluate their type, their existence and their relationships. Relationships were classified as dependent or sporadic. A dependent relationship required that one factor's influence be dependent on the existence of the other factor and was represented by a solid line connecting the two factors. A sporadic relationship required that dependency occurred in only certain circumstances and was represented by a dashed line connecting the two factors.

As the objective of this research was to investigate the influence of stakeholder 
communication on the consensus process the element 'consensus making process' was represented as the pinnacle of the IC Model. Consensus requires communication to occur therefore a dependent relationship between these two factors was obvious. The type of consensus, i.e. natural or forced, is dependent on the presence or lack of presence of negotiation and negotiation requires communication therefore a dependent relationship was illustrated between these three factors. The sporadic relationship between consensus and conflict was also obvious as achieving consensus does not necessarily involve conflict. Again to resolve conflict, negotiation is not necessarily required as a resolution can be dictated rather than achieved therefore the sporadic relationship between conflict and negotiation was apparent. There were further indications from the literature that the relationships between power and communication and again between power and trust were sporadic as none of these were dependent on each other for existence. However, there was also evidence that trust could not exist without knowledge and therefore they shared a dependent relationship. Common sense indicated that cooperation, collaboration and compromise would be dependent on communication as all of these factors required communication to occur. It was further demonstrated that negotiation was dependent on cooperation, collaboration and compromise as it requires all three to be facilitated. Also obvious were the relationships between intersubjectivity, corporate culture and communication. It is common knowledge that an organisations culture cannot exist without communication whether that be verbal or non verbal therefore this indicated a dependent relationship. It is also common knowledge that every communication that occurs, including communication of the corporate culture is subject to an individual's intersubjectivity thus indicating dependent relationships.

\section{RESEARCH APPROACH}

As the proposed model of consensus was developed primarily through the hermeneutic interpretation of literature (Giddons 1991), it was then necessary to formally evaluate the model, which called upon the second cycle of hermeneutic interpretation as based on the exploratory empirical work. This research involved the elicitation of people's knowledge and experience, research of which (Bovee \& Thill 2000, Carlsson 2002) could effectively involve Interpretivist epistemology which acknowledges that understanding of the social world can be gained from participant's perspective (Khazanchi \& Munkvold 2003).

A single case study of one organisation was used to facilitate data collection to improve our understanding of the factors and their relationships. The method's adoption was motivated by the Yin's argument (Yin 1994, 1993) that the relative size of a sample does not improve the research findings if the parameters established for the research are met. It was decided that semi-structured, face to face, one on one interviews with the individual stakeholders ${ }^{17}$ of the case study would be the most efficient and effective technique to provide rich data. Representative sampling was used to find an organisation that had or was in the process of developing software requirements for an information system. The selected project had all stakeholders within one office and was nearly complete. The software requirements were being produced for the development of an interactive Website for use by the organisation's existing clientele. It was to facilitate client access to their account details and other general organisation information. Five stakeholders from the project group were interviewed, who

\footnotetext{
${ }^{17}$ Developers of the specification will also be enveloped under the title stakeholder.
} 
were representative of the type of participants usually involved in the systems development process (Yin 1993).

The case study rich data was evaluated using the qualitative method as we had to facilitate collection and interpretation of data that would arise from stakeholder's memory of events rather than observation and it was deemed highly unlikely that the participants would directly refer to all factors (Yin 1993). Additionally the level of influence of each factor was to be established based on stakeholder factual and emotional responses and these responses, which called upon qualitative analysis (Coombes 2001), and so quantitative methods have not been considered for this research. An inductive approach was used to establish a model that might be useful as a tool for understanding consensus in general, the focus of the investigated social phenomena (Hancock 2002).

In keeping with the principles of hermeneutics, and to improve the richness of data, a third cycle was performed. Following the establishment of the model of factors from the data collected through the case study two focus groups were convened. The first focus group, as consisting of three stakeholder types, were deemed representative of the stakeholders involved in the production of software requirements. This included a project manager, a developer and an end user. This was also the case with the second group of five people who were developers and project managers and all had experience as end users. To seek opinions from a diverse experience base the other criterion was:

- That all participants to the focus group must have had some involvement in the production of more than one set of software requirements;

- That none were to be associated with the original case study organisation; and

- None should be working together.

Prior to the meetings each participant was provided with a definition of the factors in the IC Model. As with the case study interviews the session was semi structured in nature. Participants were informed at the beginning of the session that they were expected to voice their agreement or disagreement with others in the group on the position and or inclusion of factors in the IC Model. They were also asked to justify any divergence with evidence based on their past experiences. The discussion of each factor in the IC Model continued until all participants reached a consensus (sic!) on the position of a factor in the hierarchy of the IC Model. From all the data collected conclusions were drawn and presented as findings.

\section{LIMITATIONS}

Due to the level of researcher interpretation of the data collected in this study and the use of a single case study with a small number of participants, the evidence for the IC Model factors and the relationships in the data should be concluded as tentative at the moment. The IC Model developed in this study can serve only as a guide for future researchers attempting examination of the various aspects of consensus as the opportunity for any one study to encounter naturally occurring situations in which formal, controlled tests of this model would be likely, appear virtually impossible.

\section{CASE STUDY FINDINGS}

Throughout the interview process the respondents discussed many issues, which support the 
notion that several factors influence the stakeholder consensus process. The factors highlighted by the interviews include: the communication and negotiation processes, conflict, power, knowledge, trust, corporate culture, intersubjectivity, cooperation, collaboration and compromise. This section reports on the shared views and concerns of the interview participants (here referred to by their code, i.e. P1, P2, ...) as evidence for the inclusion of each factor in the model, verification of each factor's position, relationships and relationship type. Finally, it culminates in the improved IC Model, which was then used for discussion by the focus group to assist in further verification of the IC Model's completeness.

In respect to the case study, nearly all respondents to the interviews, when asked about their involvement in conflict during the consensus process, denied the existence of any conflict. Some participants (such as P5) claimed not to come into contact with work-related conflict at all. However there was also an indication (by P1 and P4) in the responses that conflict was occurring but may be suppressed, not recognised or purposely not communicated. Some (P1) also noted that the project group tried to avoid conflict but when it had happened the conflict was resolved by the adjustment of one party's opinion. Furthermore, an acknowledgement was made (by P4) that a breakdown in communication between the factions of the project group actually occurred. Although negotiation did not appear to be a major factor in our case study, the established models of group dynamics seem adamant that negotiation is always a major part of the consensus process and therefore should be considered an influence on it.

While the literature acknowledged the communication process as another factor of influence on the consensus process, it also acknowledged that through communication, stakeholder actions could influence the consensus process. This was evidenced in the participants' responses.

Throughout the study it became apparent that the project team featured two distinct factions. There also appeared to be a disparity between the two factions perception as to the amount of communication occurring. Most respondents reported that open communication occurred, however the majority indicated that this happened within the hierarchal levels of the project group and not between the levels. Only one respondent (P1) felt that communication was open and flowing between the levels.

Power comes in many forms including 'minority and group power' or perceived and actual power. The responses in this study have shown that many forms of power had a significant influence on consensus. They also show that the power influence can have either detrimental or beneficial effect on the project. All respondents have been either a perpetrator or recipient of an exertion of power and in some cases both. All respondents acknowledged the influence of power on the consensus process in their answers. All admitted to either changing a decision or seeing others change their decision based on the influence of some form of power whether that be power of the personality, group power, power of someone's social or employment position.

The responses provide evidence that power is an intrinsic element of the IC Model as a factor of influence on the consensus process. Power does need to be communicated for it to have an influence on the consensus process. Even perceived power is communicated in some way therefore a relationship will be shown between power and communication. As power's influence on consensus is dependent on communication the relationship type will be dependent.

A number of participants mentioned trust to be clearly associated with knowledge (P1, P3 and P5), both being of considerable importance to the consensus formation. In most 
observed cases, trust of an employee required some prior awareness of another person's knowledge and their ability to make decisions, therefore the IC Model amalgamates trust and knowledge as a joint factor, which could also be construed as sub factors of power.

Intersubjectivity encompasses people's shared perspective, perception and interpretation, as these all contribute to shared understanding. The responses indicated that perspectives have a large influence on the consensus process. Participants have indicated that their perspectives have changed the intersubjectivity of the group or that they have gained new intersubjectivity based on others perspective. It was acknowledged earlier that people distort perception to make things fit into their patterns of perception and that this can inhibit communication. Therefore shared perception or intersubjectivity influences consensus by maintaining the flow of communication and will remain in the IC Model. Intersubjectivity can inhibit or improve communication but communication adds to the creation of intersubjectivity so a relationship exists between intersubjectivity and the communication process. Intersubjectivity only has influence on consensus if it is shared. To be shared it must be expressed which cannot occur without communication therefore it has no direct relationship with consensus but does with communication. This relationship type was later shown of casual importance.

Interestingly, we have observed very little mention of the impact of corporate culture on consensus processes. There appears, however, to be two different opinions on the corporate culture in general. All respondents approached talking about this factor with extreme caution. The respondents did not raise this subject directly and when asked if they felt the corporate culture has any influence over their meetings the answers were often guarded or not forthcoming at all. A few respondents felt they could not comment. One observation from the data was that, in general, this topic made the people interviewed uncomfortable.

In fact, only two respondents (P3 and P4) reported any direct influence of corporate culture on their work at all, not to mention the consensus. In fact, the majority of participants stated quite categorically that they felt corporate culture contributed more to a forced consensus rather than a natural one. One (P4) implied that corporate culture contributes in some ways to the increase in the level of communication and the possible success of the project. Yet, another (P3) felt that corporate culture influences budget and IT decisions rather than department segmentation.

While it is not possible to fully assess this situation, it is likely that the corporate culture was contributing to the divide between the two factions. It was previously acknowledged that an organisation's communication climate is a reflection of its corporate culture. An alternative view is that the perception of the corporate culture could be a result of the lack of communication, which may well be an oversight as opposed to a direct intention. It appears that either of these situations may be occurring in this organisation therefore corporate culture will remain in the IC Model as a factor of influence on the consensus process. Clearly a relationship exists between communication and corporate culture. Due to the influence these factors have over each other the relationship type will be classified as dependent. However it must be pointed out that there is no direct relationship between corporate culture and consensus without communication.

The interdependence between collaboration, cooperation and compromise (CCC) was not demonstrated in a direct way, however, most interviewed respondents felt that they compromised, collaborated or cooperated in some way throughout the consensus process. To our surprise, the case study data hinted that in the IC Model, CCC should be a sub factor of power rather than the communication process as it was presented in the literature. Some participants (P5) felt that they compromised their position by backing down on their 
opinion. Others (P1 and P2) linked cooperation with their ability to change their mind to agree with others. These comments have demonstrated that compromise and cooperation involve a relinquishing of power. Participants (P1 and P5) acknowledged collaboration to involve acquisition of certain results, which demonstrates that collaboration requires the establishment or strengthening of power. For this reason CCC will be represented as having a relationship to power in the IC Model. The relationship type will be presented as dependent because $\mathrm{CCC}$ all required a shift in power.

As there was very little literature on the influence of familiarity between people on consensus, it was found [22], however indirectly, that familiarity could alter the perceptions of power that is normally associated with social status or personality. This can in turn increase the alignment of intersubjectivity and alter the way people communicate by means of changing language style and tone, or by reducing the level of formality. Familiarity can further assist in the avoidance of conflict but it could also create it. Has it not been said that familiarity creates contempt; it can create a shared knowledge that results in the inducement or dissolution of trust. Familiarity has also been known to aid or hinder in negotiation.

It was quite surprising to us that familiarity was not mentioned by many of our participants as a factor of influence on consensus. Seemingly, only one respondent (P2) raised the issue of familiarity as a factor of importance to consensus. However, after careful examination of transcripts it became evident that yet another participant had implicitly referred to the feeling of 'being at ease' with their peers during discussion and communication (P5), which can be construed as a facet of familiarity.

It was finally surmised that familiarity can be considered to be part of corporate culture. Yet we must acknowledge that in IS projects frequently not all parties to the consensus process come from the same organisation, therefore it may take considerable time for these disparate stakeholders to perceive culture as having any influence on their work. However, any familiarity external parties have established with other participants, prior to the consensus process beginning, will influence them from the onset of the process. It was also surmised that intersubjectivity and familiarity could be the same factor as both are based on shared experiences and have a strong dependence on communication. At this point we decided to treat the two as separate factors and include familiarity in the IC Model as a factor of influence on consensus so it could be further examined through the focus group. This prompted a need for further investigation so familiarity was presented in the IC Model as having a relationship with consensus and communication based on its ability to influence all other factors through communication. The relationship type with communication would be dependent as it requires communication to have influence and its relationship type with consensus would be casual as consensus does not require familiarity to occur.

\section{Focus Groups Findings}

During the focus groups a new IC Model was produced. At the final stage of the meetings all respondents in the groups agreed that the initial IC Model (see Figure 1) represented their views on the factors based on their experiences. The researchers took notes on this process for later analysis. The following section discusses responses from the focus groups, which was eventually used to derive the final IC Model (see Figure 2).

The focus groups agreed with the model positions of the factors; familiarity, conflict, the communication process, knowledge and trust, CCC and power. However, in general, the groups did not agree with the positions of intersubjectivity, corporate culture and the negotiation process. 
Figure 2 - The Final IC Model (As resulted from the first focus group.)

(Note that sporadic relationships have been removed from the model.)

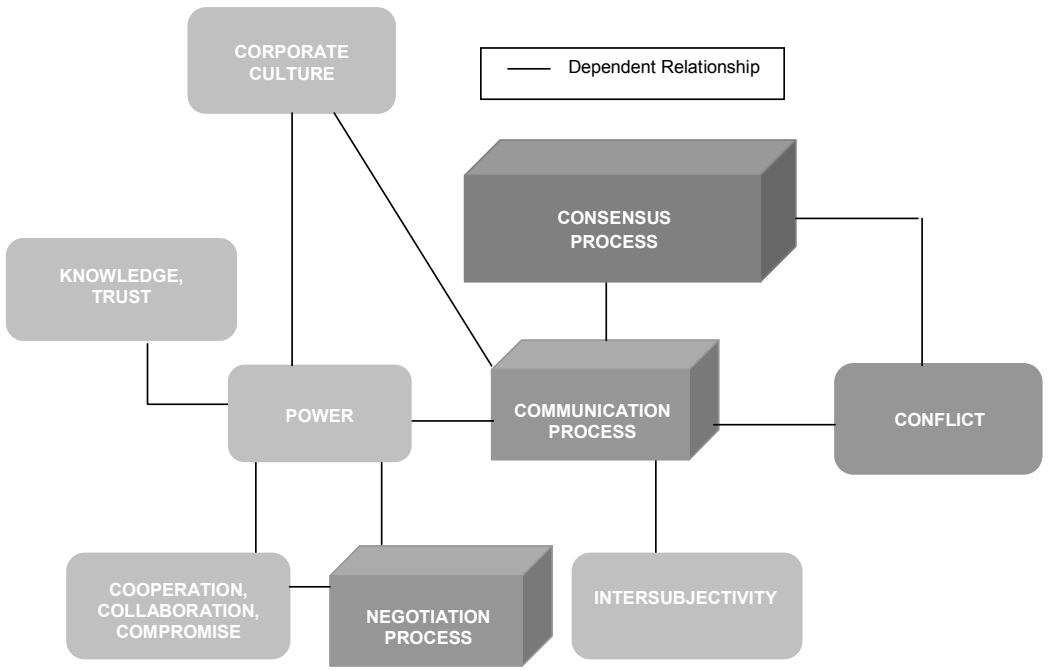

Negotiation and Power

Both focus groups agreed the negotiation process has an association with power. Several members of group 2 raised the point that it is impossible to negotiate without encountering some form of power. Both groups also agreed that negotiation should be presented at a lower level of the model than power because it has the ability to diffuse all types of power.

\section{Corporate Culture and Power}

There was unanimous concurrence that corporate culture should be presented at a lower level in the hierarchy than power. In group 2 one participant felt that without a power base you would not have a corporate culture because it is established through a demonstration or lack of demonstration of power. In addition, in both groups, some members made statement to the fact that they had seen strong corporate culture suppressing the power of subordinate or weaker stakeholders. Therefore, they thought the relationship between the two factors should be presented as dependent.

\section{Intersubjectivity and Consensus}

There was clear agreement between both groups that intersubjectivity should be a direct influence on the consensus process and therefore should have its status raised in the hierarchy. This belief was based on the opinion that every single stakeholder who enters the elicitation process comes with a preconceived opinion on every aspect of their own reality. The groups generally thought that this opinion may change with an increase in knowledge but intersubjectivity will still influence the consensus process from its commencement. In 
fact several participants from group 2 noted witnessing allegiances based on intersubjectivity that had enhanced the consensus process through resolution of conflict. However they also remarked on witnessing allegiances based on intersubjectivity that were used to hinder the consensus process by creating power bases.

IC Model Revision

Literature and the case study data were examined to assess the focus groups' decision on the positioning of 'corporate culture' in the IC Model. As earlier it was clearly stated that an organisation's communication climate is a reflection of its corporate culture and that this affects the quantity and quality of the information passing through the organisation. Alternatively communication is required to convey the corporate culture. Therefore a direct, dependent connection will be maintained between the factors corporate culture and communication process in the model. However, we were also made aware that the communication process and hence corporate culture is influenced by authoritarian management styles, which is a form of power. This supported the focus groups' argument that corporate culture is determined by the levels of power demonstrated power.

Another interesting point is in the case study in support of this idea was raised by P5. When asked about the influence of corporate culture on the group, P5 made comment that they are expected to accept all decisions and hence feel party to a forced consensus. This also demonstrates an association between power and corporate culture. Therefore corporate culture will be represented as having a direct dependent relationship with power in the IC Model.

The second factor in question was intersubjectivity. Initially one member of the group 1 thought that intersubjectivity should only be represented as having a relationship with the communication process and hence should be lower than communication in the hierarchy. However, after much discussion both focus groups believed that every stakeholder enters the consensus process with preconceived perceptions on every issue to be raised, every person involved and the outcome they desire. They acknowledged that perception is a part of intersubjectivity and hence it would influence every other factor in some way. Therefore intersubjectivity should hold the highest ranking in the hierarchy directly below consensus. It had already been established that intersubjectivity influences every aspect of our existence so there was no argument on that point. However, it was important to note that the influence comes from the communication of the intersubjectivity and not the intersubjectivity itself. This point was also acknowledged by the end user in the focus group 1. For this reason intersubjectivity is presented as a sub factor of communication with a dependent relationship (see Figure 2).

A further factor to discuss is the negotiation process. In general, the groups agreed that negotiation results in a loss or gain of power for all parties to the process. Both focus groups also thought that the negotiation process requires CCC to work. Not surprisingly, it had previously been noted that negotiation depends on compromise, which requires cooperation. Therefore the association between negotiation and CCC will be presented in the IC Model. As negotiation can't occur without CCC the relationship will be presented as dependent. The argument that negotiation has an association with power, as it requires the increase or decrease of people's power has also previously been discussed by other authors $[41,42]$. These authors' arguments substantiate the suggestion made by both focus groups, to associate negotiation with power. Hence the relationship will be portrayed as dependent, based on the knowledge that any negotiation involves a loss or gain of power.

The final factor to discuss is familiarity. The groups believed that familiarity and intersubjectivity have the same influence on the consensus process and therefore should be 
in the same level. What was interesting to note from this discussion was that both focus groups generally felt that intersubjectivity and familiarity had the same influence on the consensus process. Supporting the thinking that familiarity is actually enveloped by intersubjectivity. For this reason familiarity was removed from the IC Model under the assumption that it is a part of intersubjectivity.

\section{CONCLUSIONS}

This study expanded our knowledge of consensus and provided the basis for further research into the role of consensus in requirements elicitation. The notion of a consensus process has also been defined and the characteristics of consensus identified. Through literature review and the subsequent empirical study, it was determined how consensus impacts the requirements elicitation process in software development. The factors of influence on the consensus making process further highlighted various communication aspects.

The resulting list of factors that influence consensus includes the communication and negotiation processes, conflict, intersubjectivity, power, knowledge, trust, corporate culture, collaboration, cooperation and compromise. The surprising discovery was that every one of these factors depends on communication for some level of influence. This result makes three important contributions. First this study emphasises the importance of controlled, yet open communication to the consensus process. Second, this study highlights the factors that influence consensus so they may be addressed on an individual basis by each organisation so that unique prescriptions may be developed to improve the elicitation of requirements in a project. Finally, this in turn contributes to the improvement of project management practices through a possibility of decreasing the project failure rates.

\section{REFERENCES}

Bachmann, R. Trust, Power and Control in Trans-Organizational Relations, in SASEconference, 1999, Madison/Wisconsin, U.S.A.

Berman, E.M. and W.B.J. Werther, Broad Based Consensus Building, International Journal of Public Sector Management, 1996. 9(3): p. 61-72.

Bustard, D.W., An Experience of Principled Negotiation in Requirements Engineering, in AWRE. 2002.

Carlsson, S. Information systems development: Participation and intersubjectivity - Is this just a matter of language and communication? in ECIS Conference. 2002. Gdansk.

Cecez-Kecmanovic, D. and M. Janson. Communicative Action Theory: An Approach to Understanding the Application of Information Systems. in ACIS, Australasian Conference on Information Systems. 1999. Wellington, New Zealand.

Cecez-Kecmanovic, D. Critical Information Systems Research: A Habermasian Approach, in Bled 9th European Conference on Information Systems. 2001. Slovenia.

Child, J. and G. Möllering, The Development of Organizational Trust in the Chinese Business Context. 2001.

Coombes, H., Research Using IT. 2001, London: Palgrave.

Curtis, B., H. Krasner, and N. Iscoe, A Field Study of the Software Design Process for Large Systems, Communications of the ACM, 1988, 31(11).

Darke, P. and G. Shanks, Managing User Viewpoints in Requirements Definition, in 8th Annual Conference on Information Systems. 1997: ACIS. 
Davis, A.M., E. Yourdon, and A.S. Zweig, Requirements Management Made Easy. 2000.

Dessein, W., Coordination through Authority vs. Consensus. 2002.

Deutsch, M., The Resolution of Conflict. 1973, New Haven: Yale University Press.

Drolet, A., R. K. Larrick, and M.W. Morris. Thinking of Others: How Perspective Taking Changes Negotiators' Aspirations and Fairness Perceptions as a Function of Negotiator Relationships, in Basic and Applied Social Psychology. 1998.

Easterbrook, S., Negotiation and the Role of the Requirements Specification, in Social Dimensions of Systems Engineering: People, processes, policies and software development. 1993, Ellis Horwood: London. p. 144 - 164.

Easterbrook, S., Resolving Requirements Conflicts with Computer-Supported Negotiation. 1994

Eisenhardt, K.M. and L.J. Bourgeois, Politics of Strategic Decision Making in Highvelocity Environments: Toward a Midrange Theory, Academy of Management Journal, 1986. 31: p. 737-770.

Farrell, H., Distrust. 2002, Ontario: Russell Sage Foundation.

Finkelstein, A. and I. Sommerville, The Viewpoints FAQ. 1994.

Fisher, R. and W. Ury, Getting to Yes: Negotiating Agreement Without Giving In. 1983, New York: Penguin Books.

Flood, P.C., et al., Chief Executive Leadership Style, Consensus Decision Making, and Top Management Team Effectiveness, European Journal of Work and Organizational Psychology, 2000. 9(3): p. 401-420.

Giddens, A., Modernity and Self-Identity. 1991, California: Stanford University Press.

Hancock, B., An Introduction to Qualitative Research, 2002, Trent Focus Group.

Kaplan, M.F. and A.M. Martin, Effects of Differential Status of Group Members on Process and Outcome of Deliberation. Group Processes \& Intergroup Relations, 1999. 2(4): p. 347-364.

Kethers, S. Capturing, Formalising and Analysing Cooperation Processes: A Case Study, in ECIS. 2002. Poland

Khazanchi, D. and B.E. Munkvold. On the Rhetoric and relevance of IS Research Paradigms; A Conceptual Framework and Some Propositions, in 36th International Conference on System Sciences. 2003. Hawaii.

Kirsch, L.J., Software Project Management: An Intergrated Perspective for an Emerging Paradigm. 2000.

Klein, H.K. and M. Meyers, A Set of Principles for Conducting and Evaluating Interpretive Field Studies in Information Systems. MIS Quarterly, 1999. 23(1): p. 67-94.

Klischewski, R., Commitments Enabling Co-operation in Distributed Information Systems Development. 2001.

Lewicki, R.L., et al., Negotiation. 4th ed. 1999, Burr Ridge: McGraw-Hill/Irwin. 552.

Mills, C.E. A Unique Angle on Sensemaking About Organisational Communication During Times of Change, in ANZCA03 Conference. 2003. Brisbane.

O'Connell, F., How to Run Successful Projects II. 1996: Prentice Hall.

Ransley, P., White Paper: A Primer on Requirements Engineering \& Management Version 0.2. 2000

Rothstein, A. and C.T. Butler, On Conflict and Consensus: A Handbook on Formal Consensus Decisionmaking. 1998, Food Not Bombs Publishing.

Sandelin, R., Working With Difficult Behaviors in Meetings, Communities Journal of Cooperative Living, 1999. 104(Fall): p. 47-50. 
Sommerville, I. and G. Kotonya, Requirements Engineering With Viewpoints, BCS/IEE Software Engineering Journal, 1996, 1(11): p. 2 - 26.

Spekman, R.E., Consensus and collaboration: norm-regulated behaviour in industrial marketing relationships. Journal of Marketing, 1996, 31(11/12): p. 832-856.

Weigers, K.E., Karl Wiegers Describes 10 Requirements Traps to Avoid, Software Testing \& Quality Engineering, 2000 January/February.

Wood, C. and T. Webb, The Human Organizational Impacts of Technology Uptake and Use, in AWRE. 2002.

Yin, R.K., Case Study Research: Design and Methods, ed. T. Oaks. 1994: SAGE Publications.

Yin, R.K., Applications of Case Study research. 1993, London: Sage Publications. 